\title{
Erratum to: Nonlinear viscoelastic characterization of bovine trabecular bone
}

\author{
Krishnagoud Manda ${ }^{1}$ (D) Robert J. Wallace ${ }^{2} \cdot$ Shuqiao Xie $^{1}$. \\ Francesc Levrero-Florencio $^{1}$. Pankaj Pankaj ${ }^{1}$
}

Published online: 25 August 2016

(C) Springer-Verlag Berlin Heidelberg 2016

\section{Erratum to: Biomech Model Mechanobiol DOI 10.1007/s10237-016-0809-y}

Unfortunately, the content in the entire second column of Table 1 was incorrectly published in the original publication of the article.

The corrected Table 1 is given below and has also been corrected in the original article.

The online version of the original article can be found under doi:10.1007/s10237-016-0809-y.

Krishnagoud Manda

k.manda@ed.ac.uk

1 School of Engineering, The University of Edinburgh, The King's Buildings, EH9 3DW Edinburgh, UK

2 Department of Orthopaedics, The University of Edinburgh, Chancellors building, EH16 4SB Edinburgh, UK 
Table 1 The nonlinear VE parameters along with linear Prony coefficients and irrecoverable strains at multiple stress levels for all 19 samples

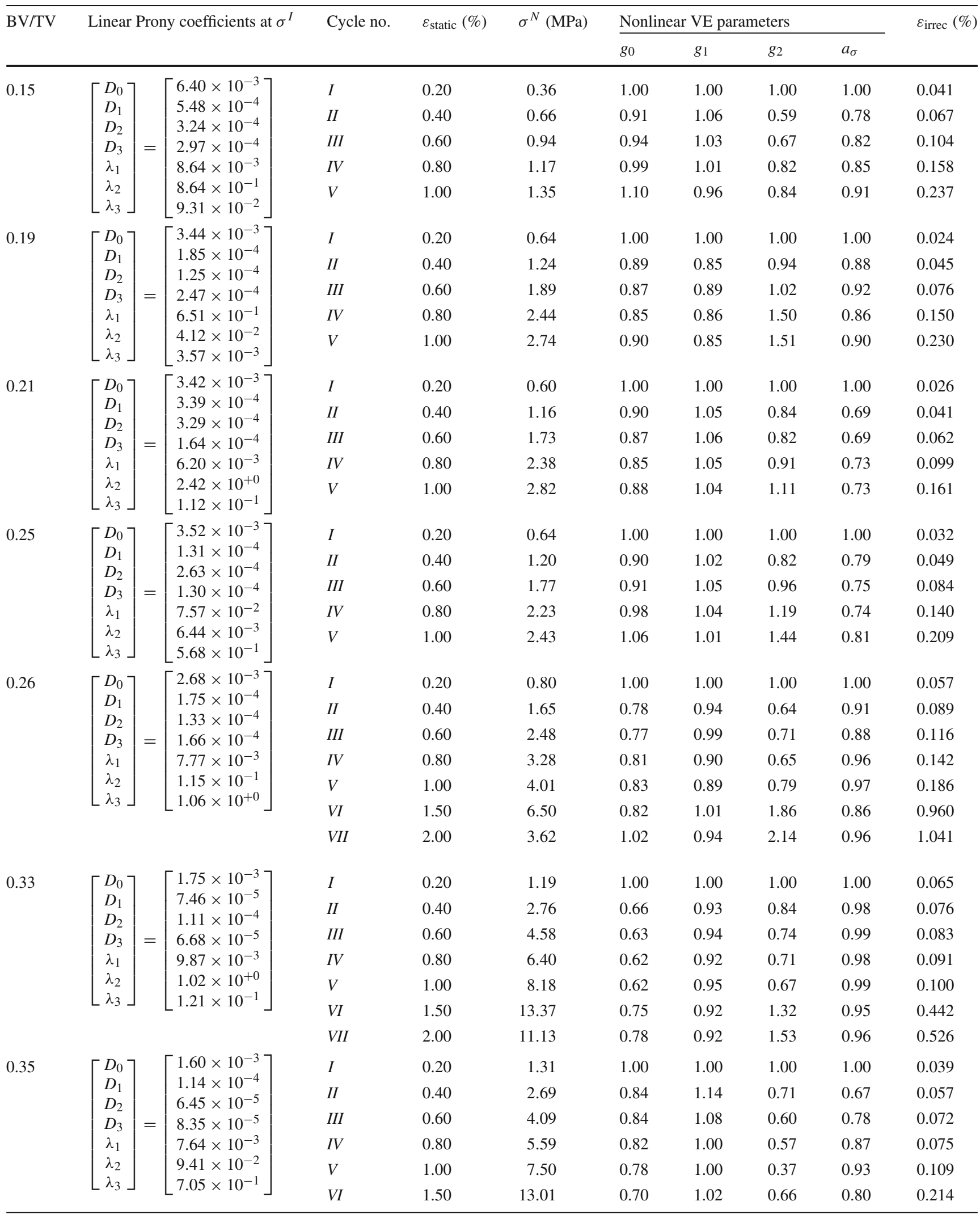


Table 1 continued

\begin{tabular}{|c|c|c|c|c|c|c|c|c|c|c|}
\hline \multirow[t]{2}{*}{$\mathrm{BV} / \mathrm{TV}$} & \multirow{2}{*}{\multicolumn{2}{|c|}{ Linear Prony coefficients at $\sigma^{I}$}} & \multirow[t]{2}{*}{ Cycle no. } & \multirow[t]{2}{*}{$\varepsilon_{\text {static }}(\%)$} & \multirow[t]{2}{*}{$\sigma^{N}(\mathrm{MPa})$} & \multicolumn{4}{|c|}{ Nonlinear VE parameters } & \multirow[t]{2}{*}{$\varepsilon_{\text {irrec }}(\%)$} \\
\hline & & & & & & $g_{0}$ & $g_{1}$ & $g_{2}$ & $a_{\sigma}$ & \\
\hline \multirow[t]{7}{*}{0.35} & \multirow{7}{*}[\begin{array}{l}{D_{0}}\\
{D_{1}}\\
{D_{2}}\\
{D_{3}}\\
{\lambda_{1}}\\
{\lambda_{2}}\\
{\lambda_{3}}\end{array}]{} & \multirow{7}{*}[\begin{array}{l}{2.16\times10^{-3}}\\
{1.41\times10^{-4}}\\
{1.43\times10^{-4}}\\
{1.11\times10^{-4}}\\
{6.41\times10^{-3}}\\
{1.41\times10^{+0}}\\
{1.22\times10^{-1}}\end{array}]{} & $I$ & 0.20 & 0.94 & 1.00 & 1.00 & 1.00 & 1.00 & 0.047 \\
\hline & & & $I I$ & 0.40 & 2.16 & 0.70 & 1.02 & 0.84 & 0.84 & 0.077 \\
\hline & & & III & 0.60 & 3.46 & 0.67 & 1.03 & 0.80 & 0.85 & 0.097 \\
\hline & & & IV & 0.80 & 4.67 & 0.65 & 1.02 & 0.75 & 0.86 & 0.118 \\
\hline & & & $V$ & 1.00 & 6.04 & 0.63 & 1.02 & 0.72 & 0.87 & 0.135 \\
\hline & & & $V I$ & 1.50 & 10.67 & 0.62 & 1.04 & 0.82 & 0.80 & 0.406 \\
\hline & & & VII & 2.00 & 11.83 & 0.62 & 1.03 & 0.94 & 0.79 & 0.522 \\
\hline \multirow[t]{7}{*}{0.36} & \multirow{7}{*}[\begin{array}{l}{D_{0}}\\
{D_{1}}\\
{D_{2}}\\
{D_{3}}\\
{\lambda_{1}}\\
{\lambda_{2}}\\
{\lambda_{3}}\end{array}]{} & \multirow{7}{*}{$=\left[\begin{array}{c}2.07 \times 10^{-3} \\
1.48 \times 10^{-4} \\
1.52 \times 10^{-4} \\
1.55 \times 10^{-4} \\
1.63 \times 10^{-1} \\
1.07 \times 10^{-2} \\
1.75 \times 10^{+0}\end{array}\right]$} & $I$ & 0.20 & 0.98 & 1.00 & 1.00 & 1.00 & 1.00 & 0.073 \\
\hline & & & II & 0.40 & 2.12 & 0.71 & 1.20 & 0.45 & 0.70 & 0.087 \\
\hline & & & $I I I$ & 0.60 & 3.67 & 0.65 & 1.02 & 0.43 & 0.87 & 0.112 \\
\hline & & & $I V$ & 0.80 & 5.28 & 0.62 & 0.95 & 0.41 & 0.93 & 0.128 \\
\hline & & & $V$ & 1.00 & 7.02 & 0.59 & 0.89 & 0.40 & 0.97 & 0.144 \\
\hline & & & $V I$ & 1.50 & 12.73 & 0.54 & 1.00 & 0.42 & 0.89 & 0.244 \\
\hline & & & $V I I$ & 2.00 & 16.68 & 0.45 & 1.01 & 0.75 & 0.79 & 0.377 \\
\hline \multirow[t]{7}{*}{0.39} & \multirow{7}{*}[\begin{array}{l}{D_{0}}\\
{D_{1}}\\
{D_{2}}\\
{D_{3}}\\
{\lambda_{1}}\\
{\lambda_{2}}\\
{\lambda_{3}}\end{array}]{} & \multirow{7}{*}{$=\left[\begin{array}{l}1.53 \times 10^{-3} \\
1.07 \times 10^{-4} \\
1.07 \times 10^{-4} \\
8.45 \times 10^{-5} \\
6.37 \times 10^{-3} \\
1.27 \times 10^{+0} \\
1.23 \times 10^{-1}\end{array}\right]$} & $I$ & 0.20 & 1.33 & 1.00 & 1.00 & 1.00 & 1.00 & 0.058 \\
\hline & & & $I I$ & 0.40 & 2.92 & 0.76 & 0.83 & 0.76 & 0.97 & 0.066 \\
\hline & & & III & 0.60 & 4.79 & 0.67 & 1.02 & 0.78 & 0.83 & 0.076 \\
\hline & & & $I V$ & 0.80 & 6.69 & 0.63 & 1.05 & 0.83 & 0.75 & 0.089 \\
\hline & & & $V$ & 1.00 & 8.53 & 0.65 & 1.07 & 0.64 & 0.79 & 0.111 \\
\hline & & & $V I$ & 1.50 & 14.81 & 0.66 & 1.02 & 0.56 & 0.86 & 0.288 \\
\hline & & & VII & 2.00 & 17.19 & 0.60 & 1.04 & 1.01 & 0.77 & 0.458 \\
\hline \multirow[t]{7}{*}{0.40} & \multirow{7}{*}[\begin{array}{l}{D_{0}}\\
{D_{1}}\\
{D_{2}}\\
{D_{3}}\\
{\lambda_{1}}\\
{\lambda_{2}}\\
{\lambda_{3}}\end{array}]{} & \multirow{7}{*}{$=\left[\begin{array}{c}2.88 \times 10^{-3} \\
2.36 \times 10^{-4} \\
5.01 \times 10^{-4} \\
2.56 \times 10^{-4} \\
1.12 \times 10^{-2} \\
2.57 \times 10^{+0} \\
1.54 \times 10^{-1}\end{array}\right]$} & $I$ & 0.20 & 0.71 & 1.00 & 1.00 & 1.00 & 1.00 & 0.127 \\
\hline & & & $I I$ & 0.40 & 1.65 & 0.46 & 0.89 & 0.51 & 0.97 & 0.170 \\
\hline & & & III & 0.60 & 2.95 & 0.44 & 0.87 & 0.41 & 0.96 & 0.201 \\
\hline & & & $I V$ & 0.80 & 4.32 & 0.43 & 0.90 & 0.40 & 0.98 & 0.220 \\
\hline & & & $V$ & 1.00 & 5.74 & 0.43 & 0.91 & 0.34 & 0.99 & 0.227 \\
\hline & & & $V I$ & 1.50 & 11.56 & 0.39 & 0.92 & 0.36 & 0.94 & 0.346 \\
\hline & & & $V I I$ & 2.00 & 14.98 & 0.39 & 0.90 & 0.33 & 0.97 & 0.491 \\
\hline \multirow[t]{7}{*}{0.40} & \multirow{7}{*}[\begin{array}{l}{D_{0}}\\
{D_{1}}\\
{D_{2}}\\
{D_{3}}\\
{\lambda_{1}}\\
{\lambda_{2}}\\
{\lambda_{3}}\end{array}]{} & \multirow{7}{*}{$=\left[\begin{array}{c}2.69 \times 10^{-3} \\
9.10 \times 10^{-5} \\
1.02 \times 10^{-4} \\
1.26 \times 10^{-4} \\
1.55 \times 10^{-1} \\
9.68 \times 10^{-3} \\
1.13 \times 10^{+0}\end{array}\right]$} & $I$ & 0.20 & 0.77 & 1.00 & 1.00 & 1.00 & 1.00 & 0.085 \\
\hline & & & II & 0.40 & 2.13 & 0.52 & 0.85 & 0.73 & 0.96 & 0.109 \\
\hline & & & III & 0.60 & 3.69 & 0.47 & 0.88 & 0.67 & 0.98 & 0.126 \\
\hline & & & $I V$ & 0.80 & 5.35 & 0.43 & 0.96 & 0.70 & 0.91 & 0.141 \\
\hline & & & $V$ & 1.00 & 7.11 & 0.43 & 0.88 & 0.60 & 0.98 & 0.160 \\
\hline & & & $V I$ & 1.50 & 13.69 & 0.37 & 0.99 & 0.69 & 0.89 & 0.295 \\
\hline & & & VII & 2.00 & 17.41 & 0.38 & 1.01 & 0.95 & 0.87 & 0.550 \\
\hline
\end{tabular}


Table 1 continued

\begin{tabular}{|c|c|c|c|c|c|c|c|c|c|c|}
\hline \multirow[t]{2}{*}{$\mathrm{BV} / \mathrm{TV}$} & \multirow{2}{*}{\multicolumn{2}{|c|}{ Linear Prony coefficients at $\sigma^{I}$}} & \multirow[t]{2}{*}{ Cycle no. } & \multirow[t]{2}{*}{$\varepsilon_{\text {static }}(\%)$} & \multirow[t]{2}{*}{$\sigma^{N}(\mathrm{MPa})$} & \multicolumn{4}{|c|}{ Nonlinear VE parameters } & \multirow[t]{2}{*}{$\varepsilon_{\text {irrec }}(\%)$} \\
\hline & & & & & & $g_{0}$ & $g_{1}$ & $g_{2}$ & $a_{\sigma}$ & \\
\hline \multirow[t]{7}{*}{0.42} & \multirow{7}{*}[\begin{array}{l}{D_{0}}\\
{D_{1}}\\
{D_{2}}\\
{D_{3}}\\
{\lambda_{1}}\\
{\lambda_{2}}\\
{\lambda_{3}}\end{array}]{} & \multirow{7}{*}{$\left.\begin{array}{l}1.47 \times 10^{-3} \\
1.09 \times 10^{-4} \\
8.72 \times 10^{-5} \\
7.91 \times 10^{-5} \\
2.81 \times 10^{+0} \\
8.63 \times 10^{-3} \\
1.76 \times 10^{-1}\end{array}\right]$} & $I$ & 0.20 & 1.37 & 1.00 & 1.00 & 1.00 & 1.00 & 0.037 \\
\hline & & & $I I$ & 0.40 & 2.97 & 0.73 & 1.03 & 0.98 & 0.83 & 0.054 \\
\hline & & & III & 0.60 & 4.74 & 0.71 & 1.04 & 0.86 & 0.82 & 0.059 \\
\hline & & & $I V$ & 0.80 & 6.57 & 0.69 & 1.04 & 0.82 & 0.84 & 0.079 \\
\hline & & & V & 1.00 & 8.44 & 0.66 & 1.03 & 0.85 & 0.85 & 0.091 \\
\hline & & & $V I$ & 1.50 & 14.45 & 0.67 & 0.91 & 0.68 & 0.96 & 0.158 \\
\hline & & & VII & 2.00 & 19.20 & 0.63 & 1.01 & 0.88 & 0.86 & 0.301 \\
\hline \multirow[t]{8}{*}{0.43} & \multirow{8}{*}[\begin{array}{l}{D_{0}}\\
{D_{1}}\\
{D_{2}}\\
{D_{3}}\\
{\lambda_{1}}\\
{\lambda_{2}}\\
{\lambda_{3}}\end{array}]{} & \multirow{8}{*}[\begin{array}{c}{1.94\times10^{-3}}\\
{1.19\times10^{-4}}\\
{1.75\times10^{-4}}\\
{9.27\times10^{-5}}\\
{7.85\times10^{-1}}\\
{7.38\times10^{-3}}\\
{9.59\times10^{-2}}\end{array}]{} & $I$ & 0.20 & 1.08 & 1.00 & 1.00 & 1.00 & 1.00 & 0.066 \\
\hline & & & $I I$ & 0.40 & 2.39 & 0.67 & 1.09 & 0.60 & 0.74 & 0.096 \\
\hline & & & III & 0.60 & 3.88 & 0.63 & 1.03 & 0.59 & 0.80 & 0.118 \\
\hline & & & $I V$ & 0.80 & 5.54 & 0.60 & 1.05 & 0.55 & 0.77 & 0.141 \\
\hline & & & $V$ & 1.00 & 7.22 & 0.61 & 0.89 & 0.52 & 0.96 & 0.146 \\
\hline & & & $V I$ & 1.50 & 13.04 & 0.57 & 1.01 & 0.42 & 0.84 & 0.268 \\
\hline & & & VII & 2.00 & 16.91 & 0.55 & 1.00 & 0.51 & 0.85 & 0.406 \\
\hline & & & VIII & 2.50 & 20.56 & 0.56 & 1.00 & 0.57 & 0.86 & 0.608 \\
\hline \multirow[t]{6}{*}{0.43} & \multirow{6}{*}[\begin{array}{l}{D_{0}}\\
{D_{1}}\\
{D_{2}}\\
{D_{3}}\\
{\lambda_{1}}\\
{\lambda_{2}}\\
{\lambda_{3}}\end{array}]{} & \multirow{6}{*}{$=\left[\begin{array}{l}9.40 \times 10^{-4} \\
3.67 \times 10^{-5} \\
6.46 \times 10^{-5} \\
6.43 \times 10^{-5} \\
1.06 \times 10^{-1} \\
6.74 \times 10^{-3} \\
9.59 \times 10^{-1}\end{array}\right]$} & $I$ & 0.20 & 2.13 & 1.00 & 1.00 & 1.00 & 1.00 & 0.042 \\
\hline & & & $I I$ & 0.40 & 4.75 & 0.74 & 1.09 & 0.70 & 0.73 & 0.057 \\
\hline & & & $I I I$ & 0.60 & 7.96 & 0.67 & 1.08 & 0.64 & 0.70 & 0.074 \\
\hline & & & $I V$ & 0.80 & 11.29 & 0.64 & 1.07 & 0.62 & 0.75 & 0.088 \\
\hline & & & $V$ & 1.00 & 14.65 & 0.61 & 1.06 & 0.68 & 0.78 & 0.102 \\
\hline & & & $V I$ & 1.50 & 24.26 & 0.66 & 1.04 & 0.75 & 0.72 & 0.180 \\
\hline \multirow[t]{6}{*}{0.46} & \multirow{6}{*}[\begin{array}{l}{D_{0}}\\
{D_{1}}\\
{D_{2}}\\
{D_{3}}\\
{\lambda_{1}}\\
{\lambda_{2}}\\
{\lambda_{3}}\end{array}]{} & \multirow{6}{*}{$=\left[\begin{array}{l}1.16 \times 10^{-3} \\
4.19 \times 10^{-5} \\
5.82 \times 10^{-5} \\
8.91 \times 10^{-5} \\
6.99 \times 10^{-2} \\
6.48 \times 10^{-3} \\
6.75 \times 10^{-1}\end{array}\right]$} & $I$ & 0.20 & 1.75 & 1.00 & 1.00 & 1.00 & 1.00 & 0.037 \\
\hline & & & $I I$ & 0.40 & 4.38 & 0.68 & 0.92 & 0.78 & 1.00 & 0.043 \\
\hline & & & III & 0.60 & 7.45 & 0.61 & 0.89 & 0.69 & 0.97 & 0.049 \\
\hline & & & $I V$ & 0.80 & 10.77 & 0.57 & 0.88 & 0.62 & 0.97 & 0.056 \\
\hline & & & V & 1.00 & 14.06 & 0.56 & 0.83 & 0.62 & 0.98 & 0.060 \\
\hline & & & $V I$ & 1.50 & 22.92 & 0.53 & 1.01 & 0.60 & 0.79 & 0.121 \\
\hline \multirow[t]{8}{*}{0.52} & \multirow{8}{*}[\begin{array}{l}{D_{0}}\\
{D_{1}}\\
{D_{2}}\\
{D_{3}}\\
{\lambda_{1}}\\
{\lambda_{2}}\\
{\lambda_{3}}\end{array}]{} & \multirow{8}{*}{$=\left[\begin{array}{l}2.03 \times 10^{-4} \\
1.60 \times 10^{-4} \\
1.50 \times 10^{+0} \\
6.85 \times 10^{-3} \\
1.29 \times 10^{-1}\end{array}\right]$} & $I$ & 0.20 & 0.89 & 1.00 & 1.00 & 1.00 & 1.00 & 0.095 \\
\hline & & & $I I$ & 0.40 & 2.25 & 0.48 & 1.13 & 0.63 & 0.66 & 0.138 \\
\hline & & & III & 0.60 & 3.87 & 0.43 & 1.09 & 0.60 & 0.69 & 0.175 \\
\hline & & & $I V$ & 0.80 & 5.62 & 0.42 & 1.08 & 0.49 & 0.74 & 0.210 \\
\hline & & & $V$ & 1.00 & 7.54 & 0.43 & 0.76 & 0.50 & 0.97 & 0.239 \\
\hline & & & $V I$ & 1.50 & 15.62 & 0.36 & 1.05 & 0.41 & 0.76 & 0.364 \\
\hline & & & $V I I$ & 2.00 & 20.88 & 0.36 & 1.03 & 0.32 & 0.82 & 0.447 \\
\hline & & & VIII & 2.50 & 26.56 & 0.33 & 1.03 & 0.53 & 0.73 & 0.656 \\
\hline
\end{tabular}


Table 1 continued

\begin{tabular}{|c|c|c|c|c|c|c|c|c|c|c|c|}
\hline \multirow[t]{2}{*}{$\mathrm{BV} / \mathrm{TV}$} & \multicolumn{3}{|c|}{ Linear Prony coefficients at $\sigma^{I}$} & \multirow[t]{2}{*}{ Cycle no. } & \multirow[t]{2}{*}{$\varepsilon_{\text {static }}(\%)$} & \multirow[t]{2}{*}{$\sigma^{N}(\mathrm{MPa})$} & \multicolumn{4}{|c|}{ Nonlinear VE parameters } & \multirow[t]{2}{*}{$\varepsilon_{\text {irrec }}(\%)$} \\
\hline & & & & & & & $g_{0}$ & $g_{1}$ & $g_{2}$ & $a_{\sigma}$ & \\
\hline \multirow[t]{7}{*}{0.53} & \multirow{7}{*}[\begin{array}{l}{D_{0}}\\
{D_{1}}\\
{D_{2}}\\
{D_{3}}\\
{\lambda_{1}}\\
{\lambda_{2}}\\
{\lambda_{3}}\end{array}]{} & \multirow{7}{*}{$=$} & \multirow{7}{*}[\begin{array}{l}{9.05\times10^{-4}}\\
{4.26\times10^{-5}}\\
{3.35\times10^{-5}}\\
{4.21\times10^{-5}}\\
{6.32\times10^{-1}}\\
{6.40\times10^{-2}}\\
{5.54\times10^{-3}}\end{array}]{} & $I$ & 0.20 & 2.22 & 1.00 & 1.00 & 1.00 & 1.00 & 0.033 \\
\hline & & & & $I I$ & 0.40 & 5.03 & 0.79 & 0.81 & 0.95 & 0.92 & 0.048 \\
\hline & & & & III & 0.60 & 8.02 & 0.75 & 0.84 & 0.88 & 0.92 & 0.059 \\
\hline & & & & $I V$ & 0.80 & 11.05 & 0.73 & 0.83 & 0.90 & 0.94 & 0.073 \\
\hline & & & & V & 1.00 & 14.10 & 0.71 & 0.87 & 0.91 & 0.96 & 0.085 \\
\hline & & & & $V I$ & 1.50 & 23.66 & 0.67 & 1.00 & 1.07 & 0.78 & 0.174 \\
\hline & & & & $V I I$ & 2.00 & 30.13 & 0.75 & 1.01 & 0.90 & 0.86 & 0.310 \\
\hline \multirow[t]{6}{*}{0.54} & \multirow{6}{*}[\begin{array}{l}{D_{0}}\\
{D_{1}}\\
{D_{2}}\\
{D_{3}}\\
{\lambda_{1}}\\
{\lambda_{2}}\\
{\lambda_{3}}\end{array}]{} & \multirow{6}{*}{\multicolumn{2}{|c|}{$=\left[\begin{array}{c}1.36 \times 10^{-3} \\
8.02 \times 10^{-5} \\
6.44 \times 10^{-5} \\
6.17 \times 10^{-5} \\
8.56 \times 10^{-1} \\
8.64 \times 10^{-3} \\
9.62 \times 10^{-2}\end{array}\right]$}} & $I$ & 0.20 & 1.49 & 1.00 & 1.00 & 1.00 & 1.00 & 0.050 \\
\hline & & & & $I I$ & 0.40 & 4.00 & 0.58 & 1.06 & 0.71 & 1.00 & 0.058 \\
\hline & & & & $I I I$ & 0.60 & 7.38 & 0.50 & 1.11 & 0.48 & 1.00 & 0.061 \\
\hline & & & & $I V$ & 0.80 & 11.01 & 0.45 & 0.90 & 0.60 & 0.98 & 0.065 \\
\hline & & & & V & 1.00 & 14.66 & 0.45 & 0.87 & 0.47 & 1.00 & 0.074 \\
\hline & & & & $V I$ & 1.50 & 24.90 & 0.42 & 0.96 & 0.49 & 0.88 & 0.129 \\
\hline
\end{tabular}

$\mathrm{BV} / \mathrm{TV}$ is the bone volume fraction, $D_{0}$ is the instantaneous compliance in $1 / \mathrm{MPa}, D_{n}(n=1,2,3)$ are transient compliance coefficients in $1 / \mathrm{MPa}$, and $\lambda_{n}(n=1,2,3)$ are reciprocal of $n$th retardation time in Prony series in $s^{-1}, \varepsilon_{\text {static }}$ is the applied static strain in each loading cycle, $\sigma^{N}$ is the stress corresponding to plateau stress in the $N$ th loading cycle in MPa. Parameters $g_{0}, g_{1}, g_{2}, a_{\sigma}$ are stress-dependent nonlinear VE parameters and $\varepsilon_{\text {irrec }}$ is the irrecoverable strain exist at the end of each loading cycle 